\title{
Effects of signal transducer and activator of transcription (STAT) genes STAT1 and STAT3 genotypic combinations on fertilization and embryonic survival rates in Holstein cattle
}

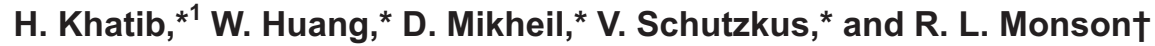 \\ *Department of Dairy Science, and \\ †Department of Animal Sciences, University of Wisconsin-Madison, Madison 53706
}

\begin{abstract}
Infertility is a major cause of dairy cow culling and economic loss. Signal transducer and activator of transcription (STAT) proteins are transcription factors that play an important role in fertility and early embryonic development, among many other functions. Previous studies have reported the association of several genes from the JAK/STAT signaling pathway with fertility traits in cattle. The STAT1 and STAT3 genes are members of this pathway and are known to interact with each other by forming a heterodimer complex that enters the nucleus and controls expression of specific genes. Thus, the objective of this study was to investigate the effects of the interactions between polymorphisms in these genes on fertilization and early embryonic survival rates using an in vitro fertilization system. A total of 7,519 oocytes, collected from 445 ovaries, were exposed to sperm and a total of 5,075 embryos were produced. Fertilization rate was calculated as the number of cleaved embryos at $48 \mathrm{~h}$ post-fertilization out of the total number of oocytes exposed to sperm. Early embryonic survival rate of embryos was calculated as the number of blastocysts on $\mathrm{d} 7$ of development out of the total number of embryos cultured. Effects of ovary genotypes on fertilization and early embryonic survival rates were evaluated. Single-SNP analysis revealed a statistically significant association between SNP25402 in STAT3 and fertilization rate. Oocytes produced from ovaries with AA genotype showed a 0.701 fertilization rate versus 0.666 and 0.663 for oocytes produced from $\mathrm{AC}$ and $\mathrm{CC}$ ovaries, respectively. The interaction between STAT3 SNP (SNP19069/SNP25402) was highly significant for survival rate but not for fertilization rate. Also, the interaction between STAT1 SNP and SNP19069 was highly significant for survival rate. Genotype combinations found to promote fertilization and embryonic survival could be incorporated into breeding
\end{abstract}

Received June 1, 2009.

Accepted September 7, 2009.

${ }^{1}$ Corresponding author: hkhatib@wisc.edu programs aimed at improving fertility performance in dairy cattle.

Key words: gene-gene interaction, survival rate, fertility, dairy cow

\section{INTRODUCTION}

Signal transducer and activator of transcription (STAT) proteins comprise a family of 7 structurally and functionally related proteins: STAT1, STAT2, STAT3, STAT4, STAT5A, STAT5B, and STAT6 (Darnell, 1997). The STAT proteins are transcription factors that play important roles in cytokine signaling pathways (Kisseleva et al., 2002). Following their phosphorylation by janus kinases (JAK), STAT proteins translocate to the nucleus to regulate transcription of different genes. The JAK/STAT pathway was found to be conserved in vertebrates (Hombría and Brown, 2002). Recent studies have shown that STAT proteins are involved in the fertilization process and in early embryonic development (Maj and Chelmonska-Soyta, 2007). Teglund et al. (1998) showed that disruption of the Stat5 gene leads to infertility in female mice as a result of small or absent corpora lutea. Truchet et al. (2004) reported that Stat1 and Stat3 are expressed in mouse oocytes and preimplantation embryos and concluded that these 2 genes might have functional importance in early embryonic development because of their roles in the cell cycle and apoptosis. Takeda et al. (1997) reported that Stat3-deficient mice die before embryonic d 8.5 and concluded that Stat3 is an essential gene for early embryonic survival and that its deficiency cannot be compensated for by other STAT proteins. Khatib et al. (2008a, 2009) showed that the CC genotype of SNP G/C in exon 8 of bovine STAT5A was associated with high fertilization and early embryonic survival rates.

Given that several genes of the JAK/STAT pathway have been found to be associated with fertility traits in cattle, STAT1 and STAT3-also members of this pathway - were chosen as candidate genes for fertilization rate and early embryonic survival in cattle. Interestingly, after their phosphorylation in the cytoplasm 
by the JAK, STAT1 and STAT3 interact with each other by forming a heterodimer complex that translocates to the nucleus and binds specific DNA sequences (Kodama et al., 1997).

The decline in reproductive performance in highproducing dairy cows is a major concern of farmers worldwide (Royal et al., 2000; Dobson et al., 2008). Major factors contributing to poor reproductive performance in dairy cattle are low fertilization rate and early embryonic loss (Santos et al., 2004; Morris and Diskin, 2008). Although genetics account for about one-third of the decline in the pregnancy rate of dairy cows (Shook, 2006), the identification of major genes affecting cow fertility has been challenging, probably because of the low accuracy of fertility data collected in the field and the low heritability of this trait. The heritability of open days and pregnancy rate for the US dairy herd is about 0.04 (VanRaden et al., 2004). To overcome these challenges, an in vitro fertilization (IVF) system has been constructed that has the advantages of a unified environment and well-isolated components of the embryonic development process. Indeed, using this system, SNP in several genes and interactions between them have been found to be associated with fertilization and early embryonic survival rates (Khatib et al., 2008a,b, 2009). Thus, the objective of this study was to investigate the single-gene effects of STAT1 and STAT3 polymorphisms and their interactions on fertilization and embryonic survival rates.

\section{MATERIALS AND METHODS}

\section{In Vitro Fertilization and Embryo Production}

Ovaries from mature Holstein cows $(\mathrm{n}=512)$ were collected from a local abattoir over a 3-yr period and immediately used in the IVF experiments. No genetic relationship information was available for these cows. Oocytes were aspirated from antral follicles and immediately incubated in maturation medium. On average, about 16.8 oocytes were aspirated from each ovary. Ovaries $(\mathrm{n}=67)$ from which fewer than 4 oocytes were harvested were excluded from the analysis. All oocytes aspirated from an ovary were combined with semen from 1 bull for an incubation period of 18 to $25 \mathrm{~h}$ as described in Khatib et al. (2008a,b). Semen of bulls (n $=12$ ) was purchased from different AI companies in Wisconsin. Fertilization rate was calculated as the number of cleaved embryos at $2 \mathrm{~d}$ post-fertilization out of the total number of oocytes exposed to sperm. After the fertilization period (fertilization $=\mathrm{d} 0$ ), putative zygotes were stripped of their cumulus cells by vortexing and were cultured until d 7 . The first morphological evaluation of embryos was done on d 5 of culture. On d
5 , embryos were evaluated for evidence of compaction or cell coalescence. Embryos undergoing compaction at this point will typically have 32 to 64 cells. On d 7 of culture, embryos previously classified as morulas (compacted) were reevaluated for the presence of a blastocoele, the presence of which classifies an embryo as a blastocyst. Survival rate of embryos was calculated as the number of normally developed blastocysts on $\mathrm{d} 7$ out of the total number of embryos cultured. Standard error of the mean of fertilization or survival rate was calculated by $\sqrt{\hat{p}(1-\hat{p}) / n}$, where $n$ was sample size and $\hat{p}$ was fertilization or survival rate. A total of 7,519 oocytes were exposed to sperm, and a total of 5,075 embryos were produced.

\section{Genotyping}

Using standard phenol/chloroform protocols, DNA was extracted from ovaries $(\mathrm{n}=445)$ and semen samples $(\mathrm{n}=12)$. The DNA concentrations were measured using a spectrophotometer (Ultraspec 2100, Amersham Biosciences, Piscataway, NJ). Three DNA pools were constructed from 50 different ovary samples to contain $50 \mathrm{ng}$ of DNA from each sample and were amplified with different sets of primers designed from the STAT3 gene, which is located on chromosome 19.

Amplification and SNP identification were as described in Khatib et al. (2008a,b). An A/G SNP was identified in exon 12 at position 19069 and an A/C SNP was identified in intron 19 at position 25402 (GenBank accession no. NC_007314; region 43752931 to 43784155). For genotyping SNP19069, the primers exon12F (5' TTCTACTTGAGCATGTACAGGG $\left.3^{\prime}\right)$ and exon12R (5' CTCTCCTGCTCAGCTATCATC $3^{\prime}$ ) were used to amplify a 589-bp fragment.

The PCR products were digested with the restriction enzyme $M S P A 1 \mathrm{I}$ at $37^{\circ} \mathrm{C}$ and then electrophoresed on a 2.0\% agarose gel. The A allele (cut) was indicated by the 384- and 205-bp fragments, and the G allele (uncut) was indicated by a single 589-bp fragment. For genotyping SNP25402, the primers intron19F (5' AACTATGTTACTTGTGGCCC $3^{\prime}$ ) and intron19R (5' AACAGATCACCAGGCTCTCC $3^{\prime}$ ) were used to amplify a 514-bp fragment. The PCR products were digested with the restriction enzyme Hinfl, which makes it possible to distinguish allele A (514-bp fragment) and allele C (167- and 347-bp fragments) when electrophoresed on a $2.0 \%$ agarose gel. The STAT1 SNP (on chromosome 2) discovery and genotyping was as described in Cobanoglu et al. (2006). Allele frequencies of SNP were calculated by counting numbers of respective alleles in the study sample. Hardy-Weinberg equilibrium was tested by chi-square test. 


\section{Statistical Analysis}

To test association between ovary genotypes for the STAT1 and STAT3 SNP and fertilization rate of oocytes or survival rate of embryos, a generalized linear model for binary response was fitted using the logit link function (McCullagh and Nelder, 1989). The linear combination of predictors can be written as

$$
\eta_{1}=b_{0}+\text { bull }+ \text { STAT5A }+ \text { ovary genotype, }
$$

where $\eta_{1}$ is the linear combination, $b_{0}$ is a constant term, bull is the effect of the semen used to fertilize the oocytes, STAT5A is the effect of the ovary genotype of an STAT5A SNP that was found to be associated with fertilization rate and embryonic survival rate in a previous study (Khatib et al., 2008a), and ovary genotype is the effect of the ovary genotype of the SNP being tested. The response variable is coded as 0 for unsuccessful fertilization or degenerate embryo or 1 for successful fertilization or normal embryo. These 2 traits were modeled for each of the 3 SNP (STAT1 SNP, STAT3 SNP25402, and STAT3 SNP19069) individually. Associations were tested using a likelihood ratio test (LRT) by comparing the above model to a reduced model missing the ovary genotype predictor, which tested whether ovary genotype had an effect on fertilization success of oocytes or survival of embryos. The LRT statistic is approximately distributed as $\chi_{\nu}^{2}$ with $\nu$ degrees of freedom that is equal to the difference in numbers of parameters of the 2 nested models compared. When sample size is large, this approximation is quite accurate (McCullagh and Nelder, 1989). The $95 \%$ confidence intervals for odds ratios (OR) between genotypes were calculated based on estimates and standard errors of the genotype term in fitted models. The most frequent homozygotes were set as reference and had OR of 1 . In addition, because of the biological interactions, 2-way interactions between the SNP were tested as described previously (Khatib et al., 2009). Briefly, the following model included both SNP and their interaction:

$$
\begin{gathered}
\eta_{2}=b_{0}+\text { bull }+ \text { STAT5A }+ \text { genotype } 1 \\
+ \text { genotype } 2+\text { genotype1:genotype } 2
\end{gathered}
$$

This model was compared with a reduced model missing the genotype1:genotype 2 term by LRT. This test was performed for each of the 3 interactions STAT1:SNP25402, STAT1:SNP19069, and SNP25402:SNP19069. Significant 2-way interactions indicate that the genotype effect of one SNP depends on the genotype of the
Table 1. Minor allele frequency (MAF) and Hardy-Weinberg equilibrium (HWE) test of SNP

\begin{tabular}{llcc}
\hline Gene/SNP & Allele & MAF & HWE $(P$-value $)$ \\
\hline STAT3/SNP19069 & A/G & 0.475 & 0.566 \\
STAT3/SNP25402 & A/C & 0.423 & $<0.001$ \\
STAT1 & C/T & 0.298 & 0.410 \\
\hline
\end{tabular}

${ }^{1}$ STAT $=$ signal transducer and activator of transcription.

interacting SNP. Therefore, fertilization or embryonic survival rates and OR were calculated for each of the 9 genotype combinations for statistically significant interactions. Statistical analysis was performed using GLM function in R (R Development Core Team, 2008) version 2.8.1, and statistical significance was claimed at the 0.01 level.

\section{RESULTS AND DISCUSSION}

Table 1 shows minor allele frequencies and tests for Hardy-Weinberg equilibrium of the 3 SNP. Only STAT3/SNP25402 showed strong evidence of disequilibrium. Table 2 shows the number of oocytes, fertilization rate, number of embryos, and survival rate for the genotypic classes of STAT1 and STAT3 SNP. Single-SNP analysis revealed a statistically significant association $(P<0.001)$ between SNP25402 in STAT3 and fertilization rate. Oocytes collected from genotype AA ovaries showed 0.701 fertilization rate versus $0.666(\mathrm{OR}=0.83$ compared with AA) and $0.663(\mathrm{OR}=0.70)$ for oocytes collected from AC and CC ovaries, respectively (Table 2). Genotypes of SNP19069 in STAT3 and the STAT1 SNP showed moderate differences in fertilization rates $(P=0.016$ and 0.014 , respectively). For embryonic survival rate, only SNP19069 showed a slight difference between STAT3 genotypes $(P=0.010)$.

To quantify the contribution of the interaction between SNP of STAT3 and STAT1, a reduced model with bull and genotype information was compared with a full model including the interactions between SNP (Khatib et al., 2009). The interaction between STAT3 SNP (SNP19069/SNP25402) was highly significant for survival rate $(P<0.001)$ but not for fertilization rate. The interaction between STAT1 SNP and SNP19069 was highly significant for survival rate $(P<0.001)$.

For significant interactions, the observed embryonic survival rates as well as OR were calculated for each genotype combination of the 2-way interactions. As expected for a statistically significant interaction, genotypic effects of one SNP were dependent on the genotype of the interacting SNP. For example, for SNP25402/SNP19069 interaction, the survival rate of embryos produced from ovaries with the AC genotype for SNP25402 (0.399) was markedly higher than that of 
Table 2. Number of oocytes, fertilization rate ( \pm SEM), number of embryos, survival rate $\left( \pm\right.$ SEM), and odds ratios ${ }^{1}(\mathrm{OR})$ for genotypic classes of signal transducer and activator of transcription (STAT) genes STAT1 and STAT3

\begin{tabular}{|c|c|c|c|c|c|c|c|}
\hline Gene/SNP & $\begin{array}{l}\text { Genotype } \\
\text { (no. of } \\
\text { ovaries) }\end{array}$ & $\begin{array}{l}\text { No. of } \\
\text { oocytes }\end{array}$ & $\begin{array}{l}\text { Fertilization } \\
\text { rate } \pm \text { SEM }\end{array}$ & OR $\left[95 \% \mathrm{CI}^{2}\right]$ & $\begin{array}{l}\text { No. of } \\
\text { embryos }\end{array}$ & $\begin{array}{l}\text { Survival rate } \\
\quad \pm \text { SEM }\end{array}$ & OR $[95 \% \mathrm{CI}]$ \\
\hline \multirow{2}{*}{ STAT3/SNP19069 } & AG (229) & 3,734 & $0.674 \pm 0.008$ & $0.93[0.82,1.05]$ & 2,518 & $0.328 \pm 0.009$ & $0.83[0.72,0.96$ \\
\hline & GG (97) & 1,641 & $0.662 \pm 0.012$ & $0.80[0.68,0.93]$ & 1,086 & $0.330 \pm 0.014$ & $0.76[0.63,0.92$ \\
\hline \multirow[t]{2}{*}{ STAT3/SNP25402 } & AA (169) & 2,772 & $0.701 \pm 0.009$ & 1 & 1,942 & $0.329 \pm 0.011$ & 1 \\
\hline & $\mathrm{AC}(155)$ & 2,697 & $0.666 \pm 0.009$ & $0.83[0.74,0.94]$ & 1,795 & $0.327 \pm 0.011$ & $0.98[0.84,1.13$ \\
\hline \multirow{2}{*}{ STAT1 } & CT (193) & 3,441 & $0.663 \pm 0.008$ & $0.86[0.78,0.96]$ & 2,282 & $0.334 \pm 0.010$ & $0.97[0.85,1.10$ \\
\hline & $\mathrm{TT}(35)$ & 540 & $0.663 \pm 0.020$ & $0.86[0.70,1.04]$ & 358 & $0.380 \pm 0.026$ & $1.16[0.92,1.48$ \\
\hline
\end{tabular}

${ }^{1}$ Odds ratios of the most frequent homozygotes were set to 1 as a reference.

${ }^{2} \mathrm{CI}=$ confidence interval.

embryos produced from AA ovaries (0.338) only when the genotype of SNP19069 was AA (OR = 1.18; Table 3). Embryos produced from AA/GG ovaries showed an extremely reduced survival rate (0.067) compared with all other genotypic combinations. However, it is worth noting that only 75 embryos were from ovaries carrying the AA/GG combination. For the STAT1/SNP19069 interaction (Table 4), the observed survival rate was 0.295 for embryos produced from CT/GG ovaries (OR $=0.63$ ) and 0.368 for embryos produced from $\mathrm{CC} / \mathrm{GG}$ ovaries; however, this difference between $\mathrm{CT}$ and $\mathrm{CC}$ ovaries for STAT1 SNP was reversed when SNP19069 genotype was AA $(0.361$ vs. $0.327, \mathrm{OR}=1.01)$. Taken together, these results strongly suggest an interaction between genes (epistasis) that interact biologically.

Truchet et al. (2004) hypothesized that because of the roles of STAT1 and STAT3 in the control of cell cycle and apoptosis, these 2 genes might have important roles in the early embryonic developmental stages. Indeed, results of this study show that single-gene associations revealed significant association of STAT3 SNP25402 and moderate association of STAT1 SNP with fertilization rate. Also, STAT3 SNP19069 was found to be associated with early embryonic survival. However, the association tests of SNP19069 with fertilization and survival rates and of STAT1 SNP with fertilization rate were moderate.

Given that STAT1 and STAT3 proteins interact biologically by forming a heterodimer and translocating from the cytoplasm to the nucleus (Kodama et al., 1997), the next logical step was to investigate the effects of the SNP-SNP interactions of these genes on fertility traits. The results showed highly significant association of STAT3 SNP (SNP19609/SNP25402; $P<$ 0.001) and of STAT1/STAT3 SNP (STAT1/SNP19069; $P<0.001)$ with early embryonic survival. Given that single-SNP analysis of STAT1 and STAT3 SNP25402 revealed only moderate significant associations with survival rate, these results support the need to include epistatic interactions in association studies (Carlborg and Haley, 2004).

For the significant interactions associated with survival rate, the observed survival rates for genotype combinations were calculated. For STAT3 SNP, the survival rates of embryos produced from AA/GG and of $\mathrm{CC} / \mathrm{AA}$ ovaries were very different compared with all other genotype combinations. Likewise, for STAT1/ SNP19069, the survival rate of embryos produced from TT/AA ovaries was relatively high compared with other genotype combinations. One can argue that the number

Table 3. Observed survival rates ( \pm SEM), number of embryos, average number of embryos per ovary, and odds ratios (OR) for genotype combinations of SNP25402 and SNP19069

\begin{tabular}{lccc}
\hline SNP25402/SNP19069 & AA $\left(\mathrm{n}^{1}, \mathrm{n}^{2}\right)$ & $\mathrm{AG}$ & $\mathrm{GG}$ \\
\hline $\mathrm{AA}$ & $0.338 \pm 0.014(1,097,12.0)$ & $0.343 \pm 0.017(747,11.5)$ & $0.067 \pm 0.029(75,6.8)$ \\
$\mathrm{OR}^{3}$ & 1 & 1 & 1 \\
$\mathrm{AC}$ & $0.399 \pm 0.033(218,14.5)$ & $0.312 \pm 0.012(1,367,11.1)$ & $0.325 \pm 0.034(188,11.8)$ \\
OR $\left[95 \% \mathrm{CI}^{4}\right]$ & $1.18[0.87,1.61]$ & $0.93[0.76,1.15]$ & $5.80[2.21,15.18]$ \\
CC & $0.627 \pm 0.063(59,14.8)$ & $0.346 \pm 0.026(338,11.6)$ & $0.359 \pm 0.017(786,11.9)$ \\
OR $[95 \% \mathrm{CI}]$ & $2.47[1.40,4.37]$ & $0.94[0.70,1.26]$ & $6.99[2.77,17.67]$ \\
\hline
\end{tabular}

\footnotetext{
${ }^{1}$ Number of embryos.

${ }^{2}$ Average number of embryos per ovary.

${ }^{3}$ Odds ratios were calculated within each genotype of SNP19069.

${ }^{4} \mathrm{CI}=$ confidence interval
} 
Table 4. Observed survival rates ( \pm SEM), number of embryos, average number of embryos per ovary, and odds ratios (OR) for genotype combinations of signal transducer and activator of transcription (STAT) gene STAT1 SNP and SNP19069

\begin{tabular}{lccc}
\hline STAT1/SNP19069 & AA $\left(\mathrm{n}^{1}, \mathrm{n}^{2}\right)$ & $\mathrm{AG}$ & $\mathrm{GG}$ \\
\hline $\mathrm{CC}$ & $0.327 \pm 0.019(587,11.1)$ & $0.319 \pm 0.013(1,261,11.1)$ & $0.368 \pm 0.020(549,12.5)$ \\
$\mathrm{OR}^{3}$ & 1 & 1 & 1 \\
$\mathrm{CT}$ & $0.361 \pm 0.018(753,13.4)$ & $0.328 \pm 0.014(1,037,11.3)$ & $0.295 \pm 0.022(440,10.7)$ \\
OR $\left[95 \% \mathrm{CI}^{4}\right]$ & $1.01[0.80,1.28]$ & $1.07[0.90,1.28]$ & $0.63[0.48,0.83]$ \\
TT & $0.508 \pm 0.063(63,12.6)$ & $0.394 \pm 0.035(198,11.0)$ & $0.268 \pm 0.045(97,8.1)$ \\
OR $[95 \% \mathrm{CI}]$ & $2.19[1.27,3.78]$ & $1.23[0.90,1.69]$ & $0.65[0.40,1.07]$ \\
\hline
\end{tabular}

${ }^{1}$ Number of embryos.

${ }^{2}$ Average number of embryos per ovary.

${ }^{3}$ Odds ratios were calculated within each genotype of SNP19069.

${ }^{4} \mathrm{CI}=$ confidence interval.

of embryos carrying these genotypes is relatively small (Tables 3 and 4). Nonetheless, for STAT3 SNP, the difference between $\mathrm{AC} / \mathrm{AA}$ and $\mathrm{AC} / \mathrm{AG}$ observed survival rate was 0.087 (Table 3). For STAT1/SNP19069 interaction, the difference between TT/AG and the TT/GG observed survival rate for genotype combinations was 0.126 (Table 4). Such differences in survival rate were not observed when comparing genotypes of individual SNP, neither could they be explained by SNP effects additively.

In a previous study, it was reported that some genes in the POU1F1 pathway did not show significant associations with fertility traits using single-gene analysis, whereas gene-gene interactions were significant (Khatib et al., 2009). The association of these genetic markers with phenotypic traits could not be revealed unless SNP-SNP interactions were taken into account. Thus, genotype combinations found to be associated with high embryonic survival rate in this study could be employed in gene-assisted selection programs aimed at improving fertility performance in dairy cattle.

The STAT1 and STAT3 genes are members of the JAK/STAT signaling pathway. Truchet et al. (2004) reported that that STAT1 and STAT3 are present in mouse oocytes and in preimplantation embryos and that the JAK/STAT pathway is functional during early embryonic development. Furthermore, previous studies have reported that several members of the JAK/ STAT pathway were found to be associated with early embryonic survival in cattle. Taken together, results of this study provide additional support for the genetic involvement of the JAK/STAT pathway in the early survival of cattle embryos.

\section{CONCLUSIONS}

In this study, the effects of $3 \mathrm{SNP}$ in the STAT1 and STAT3 genes and their interactions on fertility traits were investigated in Holstein cattle. To mimic pregnancy evaluation in live cows, an IVF system was constructed that enabled the evaluation of fertilization success and early embryonic survival, 2 initial components of pregnancy. It is conceivable to conclude that interactions between SNP in the STAT3 gene and interactions between the STAT1 and STAT3 genes contribute significantly to the phenotypic variation in embryonic survival in cattle.

\section{ACKNOWLEDGMENTS}

This study was supported by USDA Hatch grants WIS-142-PRJ16JH and WIS-142-PRJ17PH from the University of Wisconsin-Madison. The authors thank Amanda Tran and Denise Kohl, both from the University of Wisconsin-Madison, for assistance with genotyping and the IVF experiments, respectively.

\section{REFERENCES}

Carlborg, O., and C. S. Haley. 2004. Epistasis: Too often neglected in complex trait studies? Nat. Rev. Genet. 5:618-625.

Cobanoglu, O., I. Zaitoun, Y. M. Chang, G. E. Shook, and H. Khatib. 2006. Effects of the signal transducer and activator of transcription 1 (STAT1) gene on milk production traits in Holstein dairy cattle. J. Dairy Sci. 89:4433-4437.

Darnell, J. E. 1997. STATs and gene regulation. Science 277:16301635.

Dobson, H., S. L. Walker, M. J. Morris, J. E. Routly, and R. F. Smith. 2008. Why is it getting more difficult to successfully artificially inseminate dairy cows? Animal 2:1104-1111.

Hombría, J. C., and S. Brown. 2002. The fertile field of Drosophila Jak/STAT signalling. Curr. Biol. 12:R569-R575.

Khatib, H., W. Huang, X. Wang, A. H. Tran, A. B. Bindrim, V. Schutzkus, R. L. Monson, and B. S. Yandell. 2009. Single gene and gene interaction effects on fertilization and embryonic survival rates in cattle. J. Dairy Sci. 92:2238-2247.

Khatib, H., C. Maltecca, R. L. Monson, V. Schutzkus, X. Wang, and J. J. Rutledge. 2008b. The fibroblast growth factor 2 gene is associated with embryonic mortality in cattle. J. Anim. Sci. 86:2063-2067.

Khatib, H., R. L. Monson, V. Schutzkus, D. M. Kohl, G. J. Rosa, and J. J. Rutledge. 2008a. Mutations in the STAT5A gene are associated with embryonic survival and milk composition in cattle. J. Dairy Sci. 91:784-793. 
Kisseleva, T., S. Bhattacharya, J. Braunstein, and C. W. Schindler. 2002. Signaling through the JAK/STAT pathway, recent advances and future challenges. Gene 285:1-24.

Kodama, H., K. Fukuda, J. Pan, S. Makino, A. Baba, S. Hori, and S. Ogawa. 1997. Leukemia inhibitory factor, a potent cardiac hypertrophic cytokine, activates the JAK/STAT pathway in rat cardiomyocytes. Circ. Res. 81:656-663.

Maj, T., and A. Chelmonska-Soyta. 2007. Pleiotropy and redundancy of STAT proteins in early pregnancy. Reprod. Domest. Anim. 42:343-353.

McCullagh, P., and J. A. Nelder. 1989. Generalized Linear Models. 2nd ed. Chapman and Hall, London, UK.

Morris, D., and M. Diskin. 2008. Effect of progesterone on embryo survival. Animal 2:1112-1119.

R Development Core Team. 2008. R: A Language and Environment for Statistical Computing. R Foundation for Statistical Computing, Vienna, Austria. www.R-project.org.

Royal, M., G. E. Mann, and A. P. Flint. 2000. Strategies for reversing the trend towards subfertility in dairy cattle. Vet. J. 160:53-60.

Santos, J. E. P. W. W. Thatcher, R. C. Chebel, R. L. A. Cerri, and K. N. Galvao. 2004. The effect of embryonic death rates in cattle on the efficacy of estrus synchronization programs. Anim. Reprod. Sci. 83:513-535.

Shook, G. E. 2006. Major advances in determining appropriate selection goals. J. Dairy Sci. 89:1349-1361.

Takeda, K., K. Noguchi, W. Shi, T. Tanaka, M. Matsumoto, N. Yoshida, T. Kishimoto, and S. Akira. 1997. Targeted disruption of the mouse Stat3 gene leads to early embryonic lethality. Proc. Natl. Acad. Sci. USA 94:3801-3804.

Teglund, S., C. McKay, E. Schuetz, J. M. van Deursen, D. Stravopodis, D. Wang, M. Brown, S. Bodner, G. Grosveld, and J. N. Ihle. 1998. Stat5a and Stat5b proteins have essential and nonessential, or redundant, roles in cytokine responses. Cell 93:841-850.

Truchet, S., M. Chebrout, C. Djediat, J. Wietzerbin, and P. Debey. 2004. Presence of permanently activated signal transducers and activators of transcription in nuclear interchromatin granules of unstimulated mouse oocytes and preimplantation embryos. Biol. Reprod. 71:1330-1339.

VanRaden, P. M., A. H. Sanders, M. E. Tooker, R. H. Miller, H. D. Norman, M. T. Kuhn, and G. R. Wiggans. 2004. Development of a national genetic evaluation for cow fertility. J. Dairy Sci. $87: 2285-2292$ 